\title{
The Coalition Structure Core is Accessible
}

Citation for published version (APA):

Kóczy, L. Á., \& Lauwers, L. (2004). The Coalition Structure Core is Accessible. Games and Economic Behavior, 48(1), 86-93. https://doi.org/10.1016/j.geb.2003.06.006

Document status and date:

Published: 01/01/2004

DOI:

10.1016/j.geb.2003.06.006

Document Version:

Publisher's PDF, also known as Version of record

\section{Please check the document version of this publication:}

- A submitted manuscript is the version of the article upon submission and before peer-review. There can be important differences between the submitted version and the official published version of record.

People interested in the research are advised to contact the author for the final version of the publication, or visit the DOI to the publisher's website.

- The final author version and the galley proof are versions of the publication after peer review.

- The final published version features the final layout of the paper including the volume, issue and page numbers.

Link to publication

\footnotetext{
General rights rights.

- You may freely distribute the URL identifying the publication in the public portal. please follow below link for the End User Agreement:

www.umlib.nl/taverne-license

Take down policy

If you believe that this document breaches copyright please contact us at:

repository@maastrichtuniversity.nl

providing details and we will investigate your claim.
}

Copyright and moral rights for the publications made accessible in the public portal are retained by the authors and/or other copyright owners and it is a condition of accessing publications that users recognise and abide by the legal requirements associated with these

- Users may download and print one copy of any publication from the public portal for the purpose of private study or research.

- You may not further distribute the material or use it for any profit-making activity or commercial gain

If the publication is distributed under the terms of Article $25 \mathrm{fa}$ of the Dutch Copyright Act, indicated by the "Taverne" license above, 


\title{
The coalition structure core is accessible
}

\author{
László Á. Kóczy* and Luc Lauwers \\ Center for Economic Studies, Catholic University Leuven, Naamsestraat 69, B-3000 Leuven, Belgium \\ Received 22 October 2001 \\ Available online 14 October 2003
}

\begin{abstract}
For each (individually rational) payoff configuration of a TU-game with a non-empty coalition structure core there exists a finite sequence of successively dominating payoff configurations that terminates in the coalition structure core. In order to obtain this result a restrictive dominance relation-which we label outsider independent—is employed.
\end{abstract}

(c) 2003 Elsevier Inc. All rights reserved.

JEL classification: $\mathrm{C} 71 ; \mathrm{C} 73$

Keywords: Coalition structure; Core-extension; Non-emptiness; Dominance

\section{Introduction}

For a TU-game in coalitional form, there are two fundamental and strongly linked problems:

(i) what coalitions will form, and

(ii) how will the members of these coalitions distribute their total worth.

We attempt to answer these questions for a certain class of games. We presuppose some bargaining process and show that the coalition structure core, provided it is non-empty, comes forward as a natural candidate for a solution.

\footnotetext{
* Corresponding author.

E-mail addresses: laszlo.koczy@econ.kuleuven.ac.be (L.Á. Kóczy), luc.lauwers@econ.kuleuven.ac.be (L. Lauwers).

URLs: http://www.econ.kuleuven.ac.be/ew/academic/econmetr/Members/koczy/, http://www.econ.kuleuven.ac.be/ew/academic/econmetr/Members/lauwers/.

0899-8256/\$ - see front matter 두 2003 Elsevier Inc. All rights reserved. doi:10.1016/j.geb.2003.06.006
} 
Consider a TU-game and some initial individually rational payoff configuration. In case some coalition $D$ could gain by acting for themselves, it can reject this initial payoff configuration and propose a second payoff configuration. As in Shenoy (1979), Sengupta and Sengupta (1994) and Greenberg (1994, p. 1326) the improving coalition $D$ becomes a member of the new coalition structure and none of the players in $D$ looses when moving towards the new payoff configuration. We impose an additional condition. The counterproposal should be outsider independent: first, the new coalition structure should contain those coalitions in the initial configuration that do not shelter deviating players; and second, these unaffected coalitions obtain the very same payoffs. Hence, in contrast to Shenoy and Sengupta and Sengupta, the deviating coalition $D$ cannot prescribe the structure and the payoffs of those coalitions that remain unaffected when the players in $D$ separate to form a coalition. ${ }^{1}$

Once a counterproposal is established, another coalition may reject this in favour of a third payoff configuration, and so forth. Apparently, this bargaining process turns the coalition structure core, if non-empty, into an accessible set of payoff configurations:

For each payoff configuration of a TU-game with a non-empty coalition structure core, there exists a finite sequence of successive 'outsider-independent' counterproposals that terminates in the coalition structure core.

In the search for a dynamic foundation of the core, already Green (1974) made an important contribution. He established a finite process of successive counterproposals that almost surely reaches the core. Later on, Wu (1977) showed the existence of a bargaining scheme that converges to the core and rephrased this result as the core is globally stable.

Finally, our result is a continuation of the work by Sengupta and Sengupta (1996). Formulated in the language of von Neumann and Morgenstern, they proved the indirect stability of the core: no payoff allocation dominates a core payoff configuration, and each payoff configuration is indirectly dominated by a core payoff configuration. We refine this stability property in two dimensions.

First, Sengupta and Sengupta (1996) (and also Green (1974) and Wu (1977)) concentrate on the core. Hence they do not tackle problem (i). They take the coalition structure to be exogenously given and assume that the grand coalition forms. We also take the coalition formation process into account and extend the stability result to the coalition structure core.

Second, we extend the dominance relation employed by Sengupta and Sengupta (1996) to a framework involving coalition structures. And here, as already explained, we motivate a restrictive dominance relation based upon the outsider-independence condition.

The next section collects preliminaries, introduces the coalition structure core, and defines outsider independent domination. Section 3 studies outsider independent dominating chains and proves our result. The coalition structure core is characterised as the smallest set of payoff configurations that satisfies this accessibility property.

\footnotetext{
${ }^{1}$ Diamantoudi and Xue (2002) also criticise this feature of the dominance relation employed by Shenoy (1979), Sengupta and Sengupta (1994).
} 


\section{Preliminaries}

We introduce the notation and define games, payoff configurations, dominance, and the coalition structure core. As we do not assume that the grand coalition forms, we use payoff configurations (in the sense of e.g. Owen, 1995, Definition XIII.1.2) instead of imputations.

Let $N=\{1,2, \ldots, n\}$ be a set of $n$ players. Non-empty subsets of $N$ are called coalitions. A partition is a set of pairwise disjoint coalitions so that their union is $N$ and represents the breaking up of the grand coalition $N$. For a partition $\mathcal{P}=\left\{C_{1}, C_{2}, \ldots, C_{m}\right\}$ and a coalition $C$, following Owen (1995, Definition XIII.1.3) we define the partners' set $P(C, \mathcal{P})$ of $C$ in $\mathcal{P}$ as the union of those coalitions in $\mathcal{P}$ that have a non-empty intersection with $C$.

A characteristic function $v: 2^{N} \backslash\{\varnothing\} \longrightarrow \mathbb{R}$ assigns a real value to each coalition. The pair $(N, v)$ is said to be a transferable utility game in characteristic function form, in short, a game. An individually rational payoff configuration - thereafter: i.r.p.-configurationof a game $(N, v)$ is a pair $(x, \mathcal{P})$ where $\mathcal{P}$ is a partition of $N$ and the real vector $x=\left(x_{1}, x_{2}, \ldots, x_{n}\right)$ listing the payoffs of each player satisfies

$$
\forall i \in N: x_{i} \geqslant v(\{i\}) \text { and } \forall C \in \mathcal{P}: x(C)=v(C),
$$

with $x(C)=\sum_{j \in C} x_{j}$. The first condition is known as individual rationality: player $i$ will cooperate to form a coalition only if his payoff $x_{i}$ exceeds the amount he would get on his own. The second condition combines feasibility and the myopic behaviour of the players. It states that the payoff vector $x$ is efficient with respect to the coalition structure $\mathcal{P}$ : each coalition in the partition $\mathcal{P}$ allocates its value among its members. I.r.p.-configurations with the same payoff vector are said to be payoff equivalent. The set of all i.r.p.-configurations is denoted by $\Omega(N, v)$. Note that $\Omega(N, v)$ is non-empty: it contains the i.r.p.-configuration in which $N$ is split up in singletons.

Definition 1 (Outsider independent domination). Let $(N, v)$ be a game and let $a=$ $(x, \mathcal{P})$ and $b=(y, \mathcal{Q})$ be two i.r.p.-configurations. Then, i.r.p.-configuration $a$ outsiderindependently dominates $b$ by $C$ if

C1: $x(C)>y(C)$ and for all $i \in C: x_{i} \geqslant y_{i}$,

C2: $\mathcal{P}$ contains $C$,

C3: (a) $\mathcal{P}$ also contains all coalitions in $\mathcal{Q}$ that do not intersect $C$, and

(b) $x_{i}=y_{i}$ for all players $i$ outside $P(C, \mathcal{Q})$.

Coalition $C$ is called the deviating coalition.

I.r.p.-configuration $a$ outsider-independently dominates $b$ if $\mathcal{P}$ contains a coalition $C$ such that $a$ outsider-independently dominates $b$ by $C$, and we abbreviate this as $a$ o.i.dominates $b$.

Our definition is a restriction of widely used concepts of domination: If only Condition $\mathrm{C} 1$ is satisfied we talk about domination at the level of payoffs, if Conditions $\mathrm{C} 1$ and $\mathrm{C} 2$ are satisfied, about domination at the level of i.r.p.-configurations. Conditions $\mathrm{C} 3 \mathrm{a}$ and $\mathrm{C} 3 \mathrm{~b}$ are referred to as the outsider independence conditions. 
The o.i.-dominance relation should be interpreted in a dynamic way. Let $a=(x, \mathcal{P})$ o.i.-dominate $b=(y, \mathcal{Q})$ by $C$. Then, if $b$ is considered as the initial i.r.p.-configuration, one can say that coalition $C$ deviates and enforces the new i.r.p. configuration $a$. Indeed, in order to obtain a higher total payoff, coalition $C$ separates from its partners (and at least one member of $C$ gets strictly better off). The players in $P(C, \mathcal{Q}) \backslash C$ become ex-partners of $C$. They may reorganise themselves and their payoffs might decrease when moving from $b$ to $a$. In the worst case, these ex-partners fall apart to singletons. Finally, the outsiders, i.e. the players not in $P(C, \mathcal{Q})$, are left untouched.

The definition clearly indicates that o.i.-domination is more restrictive than domination at the level of i.r.p.-configurations, which was employed by Shenoy (1979) and Sengupta and Sengupta (1994) among others and where the deviating coalition is allowed to affect the payoffs of all the players and thus to ignore the behaviour and the motivation of the outsiders. The use of o.i.-domination removes these privileges.

The o.i.-dominance relation also models a merger or a breaking up. In the former case, the deviating coalition is the union of some of the coalitions in the initial partition. In the latter case, an initial coalition is split up into two or more subcoalitions; each subcoalition that is better off in the new i.r.p.-configuration can be considered as the deviating coalition.

Sengupta and Sengupta (1996) restrict their attention to the core, that is, they assume that the grand coalition forms. As a consequence, they employ the dominance relation at the level of payoff vectors. In contrast, we are also concerned with the coalition formation process. We believe that in the context of coalition formation, the o.i.-dominance relation is a natural and appropriate extension.

Now we repeat the definition of the coalition structure core, and we explain its relation with the different domination relations.

Definition 2. Let $(N, v)$ be a game and let $\Omega(N, v)$ be the set of i.r.p.-configurations. The coalition structure core-abbreviated as c.s.-core-C $(N, v)$ is the set of i.r.p.-configurations $(x, \mathcal{P})$ that satisfy coalitional rationality: for each coalition $S$ we have $x(S) \geqslant v(S)$.

Balancedness conditions in order to check whether or not the c.s.-core is non-empty are well known (see, e.g., Greenberg, 1994).

Proposition. The c.s.-core is insensitive to the domination concept used.

Proof. Consider a game with a non-empty c.s.-core and with a maximal total payoff $v^{*}=\max _{\mathcal{Q} \in \Pi} \sum_{C \in \mathcal{Q}} v(C)$, where $\Pi$ is the set of partitions of $N$. Each i.r.p.-configuration $(x, \mathcal{P})$ in the c.s.-core satisfies $x(N)=v^{*}$. On the other hand an i.r.p.-configuration $b=(y, \mathcal{Q})$ does not belong to the coalition structure core as soon as soon there exists coalition $D$ such that $y(D)<v(D)$. Such a blocking coalition $D$ has an incentive to deviate and is able to propose an i.r.p.-configuration $a=(x, \mathcal{P})$ (with $D$ in $\mathcal{P}$ ) that o.i.-dominates $b$ and therefore dominates it also on the level of i.r.p.-configurations. The set of i.r.p.configurations that are not o.i.-dominated, the set of i.r.p.-configurations that are not dominated at the level of i.r.p.-configurations and the coalition structure core all three coincide.

The same arguments hold in case the grand coalition forms and the core is non-empty. An i.r.p.-configuration $(y,\{N\})$ does not belong to the core as soon it can be blocked by 
some coalition $D$. Again, this blocking coalition $D$ can propose an i.r.p.-configuration that dominates it at the level of i.r.p.-configurations or outsider independently. In addition, $D$ can propose a new i.r.p.-configuration $(x,\{N\})$ such that $x$ dominates $y$ by $D$ at the level of payoffs.

The c.s.-core might contain payoff equivalent i.r.p.-configurations. In case the grand coalition forms, the c.s.-core includes the core.

\section{The coalition structure core is accessible}

Consider an initial i.r.p.-configuration. If a coalition can obtain a higher payoff, it is allowed to deviate (respecting the outsider independence conditions) and to propose a second i.r.p.-configuration, and so forth. This bargaining process gives rise to an o.i.dominating sequence. We show that for each i.r.p.-configuration there exists an o.i.dominating sequence that terminates in the c.s.-core. Let $(N, v)$ be a game and let $\Omega=$ $\Omega(N, v)$ be the set of all i.r.p.-configurations.

Definition 3. Let $a, b \in \Omega$. I.r.p.-configuration $a$ is said to be accessible from $b$, and we write $a \leftarrow b$ (or $b \rightarrow a$ ), if

(i) $a$ and $b$ are payoff equivalent, or

(ii) a sequentially o.i.-dominates $b$, i.e. there exists a positive integer $k$ and a sequence of i.r.p.-configurations

$$
a_{0}=b, a_{1}, \ldots, a_{k-1}, a_{k}=a
$$

such that $a_{i}$ o.i.-dominates $a_{i-1}$ for $i=1,2, \ldots, k$. The integer $k$ is said to be the length of (or the number of steps in) the o.i.-dominating sequence.

The relation ' $\leftarrow$ ' describes a possible succession of transitions from an i.r.p.-configuration to another one. We are interested in the i.r.p.-configurations that appear at the end of these sequences.

Definition 4. Let $\Delta$ be a set of i.r.p.-configurations. Then, $\Delta$ is accessible if for each $b$ in $\Omega$ there exists an $a$ in $\Delta$ such that $a \leftarrow b$.

Our main result is a characterisation of the c.s.-core.

Theorem. The coalition structure core of a game, if non-empty, is the smallest (for inclusion) set of i.r.p.-configurations that is accessible.

The proof requires the following lemma.

Lemma. Let $(N, v)$ be a game with a non-empty c.s.-core. Then, the c.s.-core is accessible. 
Proof. Let $b_{0}=\left(y_{0}, \mathcal{Q}_{0}\right)$ be an i.r.p.-configuration that is not in $C(N, v)$. In case $b_{0}$ is o.i.-dominated by an i.r.p.-configuration in $C(N, v)$, the proof is done. In case no i.r.p.configuration in $C(N, v)$ o.i.-dominates $b_{0}$, we construct an o.i.-dominating sequence that terminates in the c.s.-core. This sequence will be denoted by $b_{0} \rightarrow b_{1} \rightarrow b_{2} \rightarrow \cdots$. As a consequence, coalitions and individual payoffs have a double subscript the first one of which refers to the position in this dominating sequence.

The proof is divided into five steps. In Step 1, we select those players that can be blamed for not being able to go to the c.s.-core in one step. We call those players 'overpaid.' In Step 2 we select a deviating coalition and in Step 3 we define an i.r.p.-configuration $b_{1}$ that o.i.-dominates $b_{0}$. In Step 4 we repeat Steps 2 and 3 and construct an o.i.-dominating sequence $b_{0}, b_{1}, b_{2}, \ldots$ In Step 5 we show that this sequence reaches a c.s.-core i.r.p.configurations after a finite number of iterations.

Step 1. Defining the set of overpaid players. Interpret $b_{0}=\left(y_{0}, \mathcal{Q}_{0}\right)$ as the initial i.r.p.configuration. Let $a=(x, \mathcal{P})$ be a c.s.-core i.r.p.-configuration. A player $i$ for which $y_{0 i}>x_{i}$ is said to be overpaid relative to $a$. Let $O\left(b_{0}, a\right)$ collect these overpaid players. Since $b_{0}$ is not dominated by $a$, the set $O\left(b_{0}, a\right)$ is non-empty.

Now, we consider the collection of c.s.-core i.r.p.-configurations that minimise the number of overpaid players. Within this collection, we look for an i.r.p.-configuration $a^{*}=$ $\left(x^{*}, \mathcal{P}^{*}\right)$ that minimises the amount overpaid $y_{0}\left(O_{0}\right)-x^{*}\left(O_{0}\right)$, where $O_{0}=O\left(b_{0}, a^{*}\right)$. We consider $a^{*}$ as a c.s.-core i.r.p.-configuration close to $b_{0}$. Since $a^{*}$ belongs to the c.s.-core, we have that $x^{*}(N)=v\left(\mathcal{P}^{*}\right)=v^{*}$.

Step 2. Selecting a deviating coalition. Since the i.r.p.-configuration $b_{0}=\left(y_{0}, \mathcal{Q}_{0}\right)$ is not in the c.s.-core, there exists at least one blocking coalition, i.e. a coalition $D$ for which $v(D)>y_{0}(D)$. We select a deviating coalition $D$ as follows. First, we inspect the coalitions in the partition $\mathcal{P}^{*}$ and we look for a blocking coalition $D$ among $\mathcal{P}^{*}$. Next, if the partition $\mathcal{P}^{*}$ does not contain a blocking coalition, then the i.r.p.-configuration $b_{0}$ is efficient with respect to $\mathcal{P}^{*}$ and satisfies $y_{0}(N)=v^{*}$. In that case we select a minimal (for inclusion) blocking coalition.

Step 3. Defining a deviating i.r.p.-configuration. In order to define the payoff vector in the deviating i.r.p.-configuration $b_{1}=\left(y_{1}, \mathcal{Q}_{1}\right)$ we consider the different types of players separately.

First, we deal with the deviating players. Since $D$ blocks $b_{0}$ and $a^{*}$ is a c.s.-core i.r.p.configuration, we know that $y_{0}(D)<v(D) \leqslant x^{*}(D)$. Let $i \in D$. The payoff $y_{1 i}$ depends upon whether or not $D$ contains overpaid players.

(1) If $D$ does not contain overpaid players, then we define

$$
y_{1 i}=y_{0 i}+\delta_{i}(D) \leqslant x_{i}^{*},
$$

with $\delta_{i}(D)$ non-negative and adding up to $\delta(D)=v(D)-y_{0}(D)$.

(2) If $D$ does contain overpaid players, then we define

$$
y_{1 i}= \begin{cases}y_{0 i}+\frac{1}{\left|D \cap O_{0}\right|}[v(D)-y(D)] & \text { in case } i \text { is overpaid, } \\ y_{0 i} & \text { in case } i \text { is not overpaid. }\end{cases}
$$


In words, the deviating coalition divides the surplus $\delta(D)=v(D)-y_{0}(D)$ among its members. The overpaid players are served first and consume the whole surplus. The nonoverpaid players experience either a status quo or an improvement.

Secondly, the ex-partners of $D$ are assumed to split up into singletons. Hence, each player $i$ in $P\left(D, \mathcal{Q}_{0}\right) \backslash D$ receives his value $v(\{i\})$ as payoff. $^{2}$

Thirdly, the outsiders remain untouched: if $i \notin P\left(D, \mathcal{Q}_{0}\right)$, then $y_{1 i}=y_{0 i}$. As such, we meet the outsider independence conditions.

In conclusion: $b_{1}$ o.i.-dominates $b_{0}$. When moving from $b_{0}$ to $b_{1}$, the overpaid expartners of $D$ become non-overpaid. In case $b_{1}$ is either a c.s.-core i.r.p.-configuration or o.i.-dominated by a c.s.-core i.r.p.-configuration, the proof is complete. Otherwise, execute the next steps.

Step 4. An iteration.

We denote the set $O\left(b_{1}, a^{*}\right)$ of overpaid players in the i.r.p.-configuration $b_{1}$ by $O_{1}$. This set $O_{1}$ is a subset of $O_{0}$. We repeat Steps 2 and 3 and we generate an o.i.-dominating sequence $b_{0} \rightarrow b_{1} \rightarrow b_{2} \rightarrow \cdots$ of i.r.p.-configurations and a corresponding sequence $O_{0} \supseteq O_{1} \supseteq O_{2} \supseteq \cdots$ of sets of overpaid players. The next step shows the finiteness of necessary iterations.

Step 5. The sequence $b_{0} \rightarrow b_{1} \rightarrow b_{2} \rightarrow \cdots$ enters the c.s.-core in a finite number of iterations.

Along this o.i.-dominating sequence the set of overpaid players finds its minimal form, denoted by $O$, after a finite number of iterations. Let $b_{s}=\left(y_{s}, \mathcal{P}_{s}\right)$ be the first i.r.p.-configuration in the sequence that satisfies $O_{s}=O$. In case the partition $\mathcal{P}_{s}$ differs from $\mathcal{P}^{*}$, we can execute some more iterations (as described in Step 4). Due to the selection criteria for the deviating coalition (Step 2) we obtain an i.r.p.-configuration $b_{t}=\left(y_{t}, \mathcal{P}_{t}\right)$ with $t \geqslant s, O_{t}=O$, which is efficient with respect to $\mathcal{P}^{*}{ }^{3}$ The i.r.p.-configuration $b=\left(y_{t}, \mathcal{P}^{*}\right)$ is payoff equivalent with $b_{t}$.

We claim that the i.r.p.-configuration $b$ is in the c.s.-core. Since $b$ and $b_{t}$ are payoffequivalent, this immediately implies that $b_{t}$ is also in the c.s.-core.

In case $O$ is empty this claim is obviously true. Hence, assume that $O$ is non-empty and that the iteration is unable to reduce it further. Denote the partners' set of the overpaid players in $\mathcal{P}^{*}$ by $B$, i.e. $B=P\left(O, \mathcal{P}^{*}\right)$, and the complement of $B$ by $A$.

Since $A$ does not contain overpaid players, we have $y_{i} \leqslant x_{i}^{*}$ for each $i$ in $A$. Since $b$ belongs to $\Omega$ and since $A$ is the union of some of the coalitions in $\mathcal{P}^{*}$, we have $y(A)=x^{*}(A)$. Therefore, the payoff vectors $y$ and $x^{*}$ restricted to $A$ coincide: $\left.y\right|_{A}=\left.x^{*}\right|_{A}$.

We complete the proof of the claim (that $b$ belongs to the c.s.-core) by contradiction. Assume the existence of a coalition $D$ that blocks the i.r.p.-configuration $b$. Since $\left.y\right|_{A}=\left.x^{*}\right|_{A}$, the coalition $D$ is not a subset of $A$. Hence $D$ intersects $B$. Let $\bar{D}=P\left(D, \mathcal{P}^{*}\right)$

\footnotetext{
2 This assumption can be relaxed. The ex-partners are allowed to reorganise themselves provided none of them is overpaid in the new i.r.p.-configuration.

3 Remember that in looking for a blocking coalition, the coalitions in $\mathcal{P}^{*}$ are the first candidates to check. Hence, for each i.r.p.-configuration that is not efficient with respect to $\mathcal{P}^{*}$, there will be an efficient i.r.p.configuration later on in the sequence.
} 
be the partners' set of $D$. Since $y$ and $x^{*}$ are efficient with respect to $\mathcal{P}^{*}$, we have $y(\bar{D})=$ $x^{*}(\bar{D})=v(\bar{D})$. Since $O$ cannot be reduced, the coalition $D$ contains all the overpaid players in $\bar{D}$. Therefore, $\bar{D} \backslash D$ only contains non-overpaid players and thus satisfies $y(\bar{D} \backslash D) \leqslant x^{*}(\bar{D} \backslash D)$. Use the efficiency of $y$ and $x^{*}$ with respect to $\mathcal{P}^{*}$ together with the fact that $a^{*}$ is a c.s.-core i.r.p.-configuration to conclude that $y(D) \geqslant x^{*}(D) \geqslant v(D)$. Hence, $D$ is not blocking. Contradiction.

Therefore, $b$ and then also $b_{t}$ belong to the c.s.-core and the o.i.-dominating sequence $b_{0}, b_{1}, \ldots, b_{t}$ enters the c.s.-core after a finite number of iterations.

In order to stress the impact of the particular construction in the above proof we give an example of a bargaining scheme that does not enter the c.s.-core.

Example. Consider a three-player game in which each singleton has value 0, each pair has value 2 , and the grand coalition has value 6 . The core is non-empty. Nevertheless, the next three i.r.p.-configurations generate a cycle of dominating i.r.p.-configurations:

$$
((1,1,0),\{1,2\},\{3\}), \quad((1,0,1),\{1,3\},\{2\}), \quad \text { and } \quad((0,1,1),\{2,3\},\{1\}) .
$$

We conclude by the proof of the characterisation of the c.s.-core.

Proof of the Theorem. Accessibility follows from the previous lemma. Furthermore, each i.r.p.-configuration in the c.s.-core is not o.i.-dominated. This implies minimality.

\section{Acknowledgments}

This paper benefited from presentations at the LGS2 Conference (St Petersburg, June 2001) and at the Warwick Summer Research Conference (July 2001). Discussions with Hideo Konishi and Licun Xue have led to a considerable improvement of the paper. We also thank Gerard van der Laan, Anne van den Nouweland, and two anonymous referees. The first author gratefully acknowledges the joint support (SOR-H/99/23, SOR-H/00/01, $\mathrm{COE} / 01 / 02$ and DB/02/18) of the Catholic University Leuven and the Soros Foundation.

\section{References}

Diamantoudi, E., Xue, L., 2002. Coalitions, agreements and efficiency. Discussion paper 2002/47. CORE, Louvain-la-Neuve.

Green, J.R., 1974. The stability of Edgeworth's recontracting process. Econometrica 42, 21-34.

Greenberg, J., 1994. Coalition structures. Chapter 37 in: Aumann, R.J., Hart, S. (Eds.). In: Handbook of Game Theory with Economic Applications, vol. II. Elsevier, Amsterdam, pp. 1305-1307.

Owen, G., 1995. Game Theory, 3rd Edition. Academic Press, San Diego.

Sengupta, A., Sengupta, K., 1994. Viable proposals. Int. Econ. Rev. 35, 347-359.

Sengupta, A., Sengupta, K., 1996. A property of the core. Games Econ. Behav. 12, 266-273.

Shenoy, P.P., 1979. On coalition formation: a game-theoretical approach. Int. J. Game Theory 8, 133-164.

Wu, L.S.-Y., 1977. A dynamic theory for the class of games with nonempty cores. SIAM J. Appl. Math. 32, 328-338. 